\title{
Conductive Composite Biosensor System for Electrochemical Indinavir Drug Detection
}

\author{
Natasha Ross, Nicolette Hendricks-Leukes, Rachel Fanelwa Ajayi, \\ Priscilla Baker, and Emmanuel I. Iwuoha
}

SensorLab, Department of Chemistry, University of Western Cape, Private Bag X17, Bellville 7535, South Africa

Correspondence should be addressed to Natasha Ross; nross@uwc.ac.za

Received 3 July 2015; Accepted 26 August 2015

Academic Editor: Josefina Pons

Copyright (C) 2015 Natasha Ross et al. This is an open access article distributed under the Creative Commons Attribution License, which permits unrestricted use, distribution, and reproduction in any medium, provided the original work is properly cited.

\begin{abstract}
Indinavir is a protease inhibitor antiretroviral (ARV) drug, which forms part of the highly active antiretroviral therapy during the treatment of HIV/AIDS. Indinavir undergoes first-pass metabolism through the cytochrome P450 (CYP) enzymes in the human liver, of which CYP3A4 is the most influential isoenzyme. Multidrug combination therapy and, as such, therapeutic drug monitoring (TDM) during HIV/AIDS treatment are therefore critical, to prevent adverse interactions. The conventional sensitive and specific assays available for quantifying ARV drugs, however, suffer from distinct disadvantages. In this regard, biosensors can be used to provide real time information on the metabolic profile of the drug. In this study, a biosensor with cobalt(III) sepulchrate trichloride $\left\{\mathrm{Co}(\mathrm{Sep})^{3+}\right\}$ as diffusional mediator was constructed. The biosensor platform consisted of CYP3A4 immobilized onto a gold nanoparticle (GNP) overoxidized polypyrrole (OvOxPpy) carrier matrix. The biosensor exhibited reversible electrochemistry, with formal potential determined as $-624 \pm 5 \mathrm{mV}$, from voltammetric analysis, with overall electron transfer being diffusion controlled. The biosensor showed typical electrocatalytic response to dioxygen $\left(\mathrm{O}_{2}\right)$, exemplified by the distinct increase in the cathodic peak current $\left(I_{p, c}\right)$. A concentration-dependent increase in $I_{p, c}$ was observed in response to consecutive additions of Indinavir.
\end{abstract}

\section{Introduction}

Antiretroviral (ARV) drugs are ubiquitously associated with treatment of HIV/AIDS [1]. These drugs work by maintaining a plasma level concentration at the virus (HIV) replication site thereby inhibiting viral mutation and viral resistance and suppressing viral load of patients [2]. Treatment is implemented through highly active antiretroviral therapy (HAART), which is a multidrug combination regimen, consisting of ARV drugs from three basic groups, including the nucleoside reverse transcriptase inhibitors (NRTIs), nonnucleoside reverse transcriptase inhibitors (NNRTIs), and protease inhibitors (PIs). The PIs, as well as the NNRTIs, undergo extensive phase I metabolism through the cytochrome P450 (CYP) enzymes present in the liver, of which the most influential isoenzyme involved is CYP3A. One of the major aspects associated with HAART treatment is adverse drug interactions and, as such [3], the need for therapeutic drug monitoring during HIV/AIDS treatment is pivotal [4]. Sensitive and specific assays available for quantifying ARV drugs are based on high performance liquid-chromatography and/or gas chromatography (HPLC and GC) with ultraviolet detection or HPLC and/or GC-mass spectrometry. These methods, however, are very complex, requiring extensive sample preparation and skilled operators, are expensive, and are not designed to give rapid analysis and real time measurements. In this regard, biosensors can be used to provide real time information on the metabolic profile of the drug necessary for modulating therapy to the patient's benefit. During the reduction of $\mathrm{P} 450$ s according to the scheme $\mathrm{Fe}^{3+}+\mathrm{e}^{-} \rightarrow \mathrm{Fe}^{2+}$, NADPH or NADH is exhausted [5]. Therefore, P450s and enzyme electrodes based on P450s are promising for the application of biosensors. However, achieving effective electrical communication between the electrode and the enzyme in the case of heme-enzymes, such as the CYP3A4 enzyme, can be problematic [6]. This is because the electrochemically active heme-centre is buried in the protein structure to gain hydrophobic environment for catalysis, thus a longer direct electron transfer distance between the prosthetic group and the electrode surface. 
Therefore, reduction in the size of enzyme carrier materials can greatly improve the efficiency of immobilized enzymes [7]. Numerous publications indicate organic conducting polymers as a convenient biosensor component, forming an appropriate environment for the immobilization of enzyme at the electrode surface [8]. Amongst the studied polymers, polypyrrole (Ppy) has attracted considerable attention. This polymer can be easily prepared by electrochemical oxidation of the monomer in the presence of suitable dopant ions. Concomitantly, overoxidation is an irreversible processes associated with loss of conductivity. However, overoxidizedpolypyrrole (OvOxPpy) is an excellent matrix material used for deposition of metal nanoclusters. Au nanoparticles (GNP), in particular, due to their unique properties such as good conductivity, useful electrocatalytic, and biocompatibility, were considered excellent integration material in the electrochemical biosensors [9]. The presence of GNPs gives immobilized protein molecules more freedom of orientation and, as such, permits proteins to orient in conformations which are more favorable for direct electron transfer [10]. The gold particles form the conducting electrode and thus the site of electron transfer when anchored to the substrate [11]. The usual methods for enzyme immobilization include direct physical adsorption [12, 13], encapsulation into hydrogel [14], cross-linking [15], and covalent binding [16]. However, a key requirement of enzyme immobilization is attachment without the bioactivity being sacrificed $[17,18]$. Here, the $\mathrm{GNP} \mid \mathrm{OvOxPpy}$ modified film provides an attractive template for enzyme immobilization due to the fast, inert low temperature process.

In this work, OvOxPpy-film [19] infused with conductive $\mathrm{Au}$ nanoparticles was constructed on a glassy carbon electrode and used as platform for CYP3A4 enzyme immobilization to fabricate a biosensor for selective and sensitive detection of Indinavir (ARV drugs) in the large presence of $\mathrm{PBS}$ at $\mathrm{pH}$ 7.41. The goal was to obtain results with precision and accuracy and at increasingly lower concentration levels of the substance being determined.

Electroanalytical Methods Applied for the Determination of Anti-HIV Drugs. Indinavir, [1(1S,2R),5(S)]-2,3,5-trideoxy-N(2,3-dihydro-2-hydroxy-1H-inden-1-yl)-5-[2-[[(1,1-dimethylethyl)-amino] carbonyl]-4-(3-pyridinylmethyl)-1-piperazinyl]-2-phenylmethyl)-D-erythro-pentonamide sulphate, is HIV protease inhibitor metabolized by the cytochrome P450 system. It has been probed by various capillary electrophoretic, spectrophotometric, and liquid chromatographic methods; however, all the reported methods are arduous and time-consuming and require highly sophisticated equipment [20]. Up to date a great deal of biosensors were developed for determination of Indinavir. However, only one paper reported anodic voltammetry using glassy carbon electrode. Ignaszak et al. [21] developed a novel therapeutic biosensor for the determination of IND. In this study, pH 6.0 Britton-Robinson buffer solution was selected for analytical medium in which IND exhibited diffusion controlled oxidative peak at $+0.89 \mathrm{~V}$. Also the peak current varied linearly with drug concentration in the range between $2.8 \times 10^{-7}$ and $5.0 \times 10^{-5} \mathrm{M}$. The constructed biosensor also showed quasi-reversible electrochemistry of the $\mathrm{Fe}^{3+} / \mathrm{Fe}^{2+}$ redox species of the heme thiolate CYP3A4 enzyme under aerobic and anaerobic conditions with a detection limit and response time of $6.158 \times 10^{-2} \mathrm{mgL}^{-1}$ and $40 \mathrm{~s}$, respectively.

\section{Experimental}

2.1. Reagents and Instruments. The gold nanoparticles were produced by the reduction of $\mathrm{Au}$ (III) involving the use of $\mathrm{HAuCl}_{4}$ in the presence of $\mathrm{KCl}$. Cytochrome P450 3A4 (CYP3A4) (Mw 52 000) was prepared by genetic engineering with subsequent purification from a human CYP3A4 cDNA clone-overexpressed in Escherichia coli (E. coli) cells, consisting of only the terminal oxidase (heme domain) prepared as $8.17 \mu \mathrm{M}$ solution. Cobalt(III) sepulchrate trichloride $\left\{\mathrm{Co}(\mathrm{Sep})^{3+}\right\}$ and serum from bovine albumin were products of Sigma-Aldrich. Dipotassium hydrogen phosphate $\left(\mathrm{K}_{2} \mathrm{HPO}_{4}\right)$ and potassium dihydrogen phosphate $\left(\mathrm{KH}_{2} \mathrm{PO}_{4}\right)$ monohydrate salts and $\mathrm{KCl}$ were purchased from SigmaAldrich. Electrolyte solutions were $50 \mathrm{mM}$ of PBS (pH 7.4), containing $100 \mathrm{mM} \mathrm{KCl}$ as the supporting electrolyte. Working solution of Indinavir was prepared from Indinavir sulphate, obtained through extraction from the capsules (Crixivan)-purchased from Merck \& Co., Inc. Ultrapure water with resistivity $18.2 \mathrm{M} \Omega \mathrm{cm}$ was prepared using Millipore Synergy water purification system. All reagents were of analytical grade. Electrochemical experiments were performed with BAS 100W electrochemical workstation and electrodes from BioAnalytical Systems, BAS, USA. Cyclic and square wave voltammograms were recorded with a computer interfaced to the BAS 100W workstation. A glassy carbon disk electrode (GCE) of area $0.071 \mathrm{~cm}^{2}$ (diameter $3 \mathrm{~mm}$ ) was used as the working electrode. A platinum wire and $\mathrm{Ag} / \mathrm{AgCl}(3 \mathrm{M}$ $\mathrm{NaCl}$ ) electrodes were used as auxiliary and reference electrodes, respectively. SEM analysis was performed on JEOL JSM-7500F Scanning Electron Microscope. All voltammetric results are reported with respect to $\mathrm{Ag} / \mathrm{AgCl}$.

2.2. Preparation of Biosensor Platform: GNP|OvOxPpy ||$G C E$. Ppy was electrochemically prepared from an aqueous solution of $0.15 \mathrm{M}$ monomer, by potentiostatic method in $0.1 \mathrm{M}$ $\mathrm{LiClO}_{4}$ supporting electrolyte. Polymerization was done at a constant potential of $+800 \mathrm{mV}$ (versus $\mathrm{Ag} / \mathrm{AgCl}$ ) for $120 \mathrm{~s}$, under argon atmosphere. The Ppy-modified GCE was then carefully rinsed with milli-Q water and subsequently placed into an electrolytic cell, containing $0.1 \mathrm{M} \mathrm{NaOH}$ and overoxidation was done at $+1000 \mathrm{mV}$. The process usually took approximately $450 \mathrm{~s}$. Gold nanoparticles were then electrodeposited from an argon degassed solution of $100 \mathrm{mM}$ $\mathrm{KCl}$, containing $0.5 \mathrm{mM} \mathrm{HAuCl}_{4}$. Electrodeposition was done potentiodynamically, by cycling the potential from 200 to $-1000 \mathrm{mV}$ at a scan rate of $50 \mathrm{mV} / \mathrm{s}$ for 15 cycles.

2.3. Preparation of CYP3A4-Based Biosensor. CYP3A4 was immobilized on the GNP|OvOxPpy||GCE by drop-coating. $5 \mu \mathrm{L}$ of $1.63 \mu \mathrm{M}$ enzyme solution, containing $2.0 \mathrm{mg}$ BSA, was carefully drop-coated onto the GNP-modified GCE. The enzyme-modified electrode was then covered with a tight 

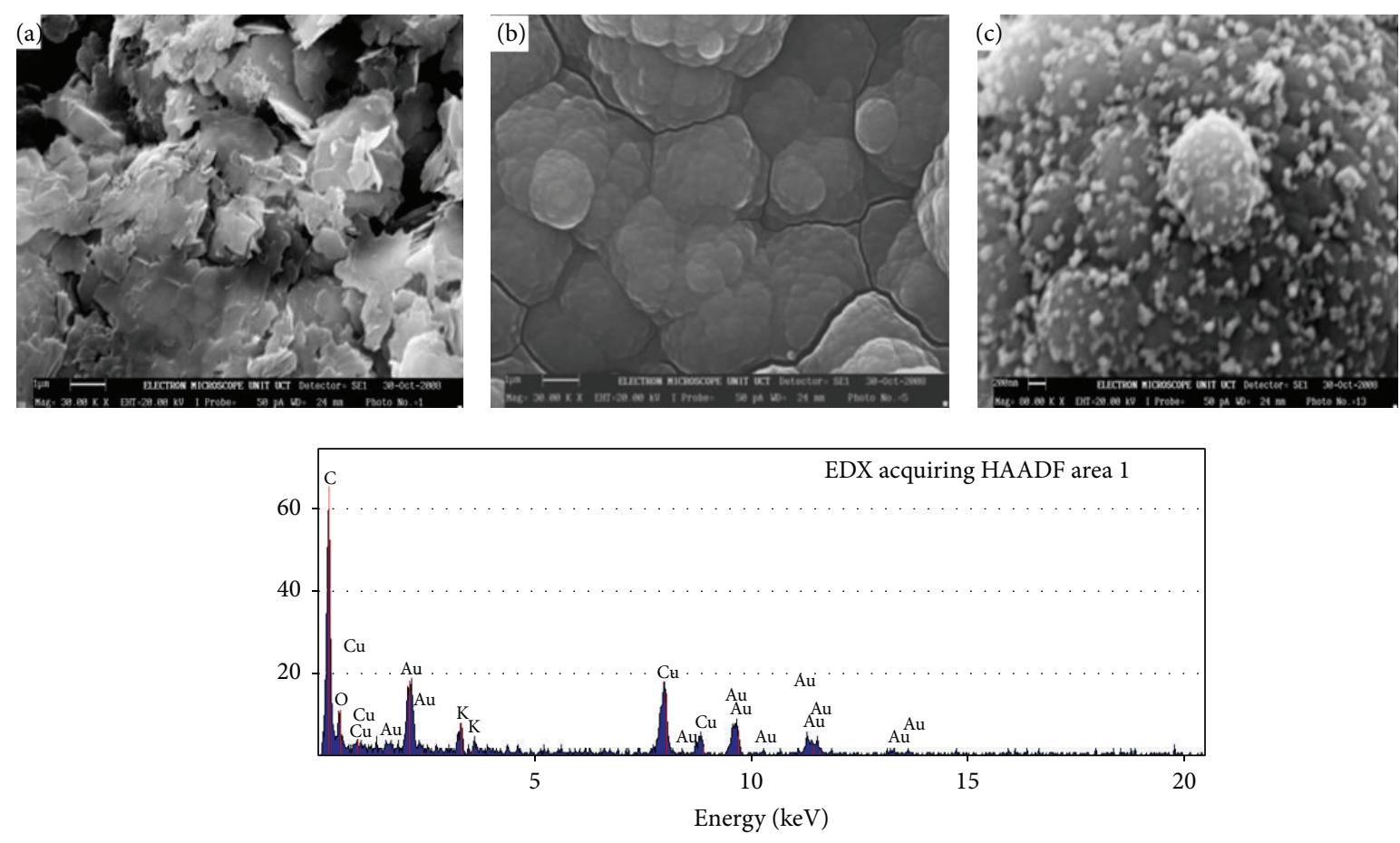

(d)

Figure 1: SEM of Ppy||GCE (a), OvOxPpy||GCE (b), and GNP|OxPpy||GCE (c), respectively, at 30000 Mag (scale = $1 \mu \mathrm{m})$ with the EDX spectrum $(d)$.

fitting lid for the first $10 \mathrm{~min}$ to form a uniform layer, after which the lid was removed and the enzyme layer was slightly dried for another $40 \mathrm{~min}$, but still retaining wet camera. This was then followed by the deposition of $4 \mu \mathrm{L}$ agarose $(1 \%$, $\mathrm{w} / \mathrm{v}$ ), after which the prepared biosensor was immediately placed at $4^{\circ} \mathrm{C}$ for at least $1 \mathrm{~h}$. The biosensor was denoted by CYP3A4|GNP|OvOxPpy||GCE.

2.4. Electrochemical Procedures. All voltammetric analysis was done at $25^{\circ} \mathrm{C}$ in the presence of $1 \mathrm{mM} \mathrm{Co}(\mathrm{Sep})^{3+}$ diffusional electron transfer mediator, dissolved in either degassed or undegassed PBS ( $\mathrm{pH}$ 7.4). Cyclic voltammograms were recorded in the presence and absence of oxygen and/or Indinavir at a potential scan rate $(\nu)$ of $10 \mathrm{mV} / \mathrm{s}$ versus $\mathrm{Ag} / \mathrm{AgCl}$, from an initial potential $E_{i}=-200 \mathrm{mV}$ to a switch potential, $E_{\lambda}=-1000 \mathrm{mV}$. Oysteryoung-type square wave voltammetry (SWV) was done at an amplitude of $75 \mathrm{mV}$ and frequency of $5 \mathrm{~Hz}$. The analysis of the CVs gave the formal potentials measured as the midpoint potential $\left(E^{\circ \prime}\right)$. The peak separation, $\Delta E_{p}$ (the difference between $E_{p, c}$ and $E_{p, a}$ ), and the anodic to cathodic peak current ratio $\left(I_{p, a} / I_{p, c}\right)$ of the biosensor responses were also determined.

\section{Results and Discussion}

3.1. Structure of Composite Polypyrrole Materials. Morphological characterization of the electrode platform during electrode assembly process was conducted by SEM analysis, as shown in Figure 1. The SEM images of the Ppy-modified GCE (a), OvOxPpy-film (b), and GNP|OvOxPpy||GCE (c) composite film were recorded at a magnification of 30000 , with a scale of $1 \mu \mathrm{m}$. The EDX spectrum (d) shows elemental analysis of a selected area on Figure 1(c). Figure 1(b) clearly shows that overoxidation affects the morphological properties of the polypyrrole film and, as such, the OvOxPpy-film exhibited a structural morphology that was significantly different from that of the conductive Ppy-film [22]. The OvOxPpy-film had a typical "cauliflower-like" structure, constituted by spherical micrograins. Prior research has established that overoxidation is initiated by hydroxyl radicals that form due to the oxidation of water, of which the overall process may be described by [23]:

$$
\begin{array}{r}
\mathrm{Ppy}+x \mathrm{HO}^{-} \longrightarrow \mathrm{OvOxPpy}+n \mathrm{e}^{-} \\
+m \mathrm{H}^{+}+\cdots
\end{array}
$$

Figure 1(c) shows the porous OvOxPpy-film to be an excellent matrix for the electrodeposition of gold nanoparticles, which had good surface coverage across the film with uniform size and diameters of approximately $100 \mathrm{~nm}$. The EDX spectrum confirms the presence of gold within the OvOxPpy matrix.

During overoxidation, a large current is passed, which decreased as oxidation progressed, eventually levelling off and fluctuating slightly around one value, which indicates the completion of the overoxidation process, as shown in Figure 2. During overoxidation, nucleophilic attack of the radical cationic pyrrole units, in the polymer by hydroxide 


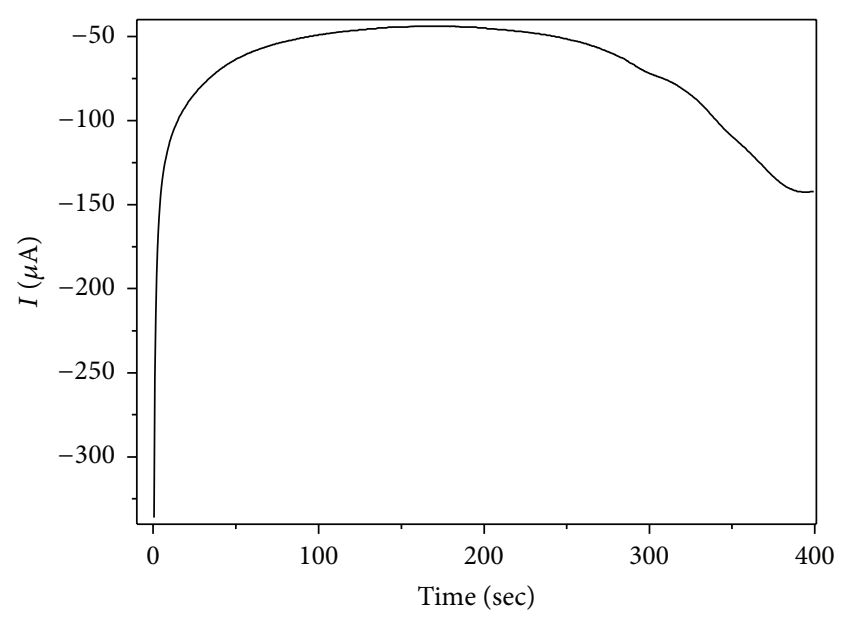

FIGURE 2: Current time curve for polypyrrole overoxidation (OvOxPpyl|GCE).

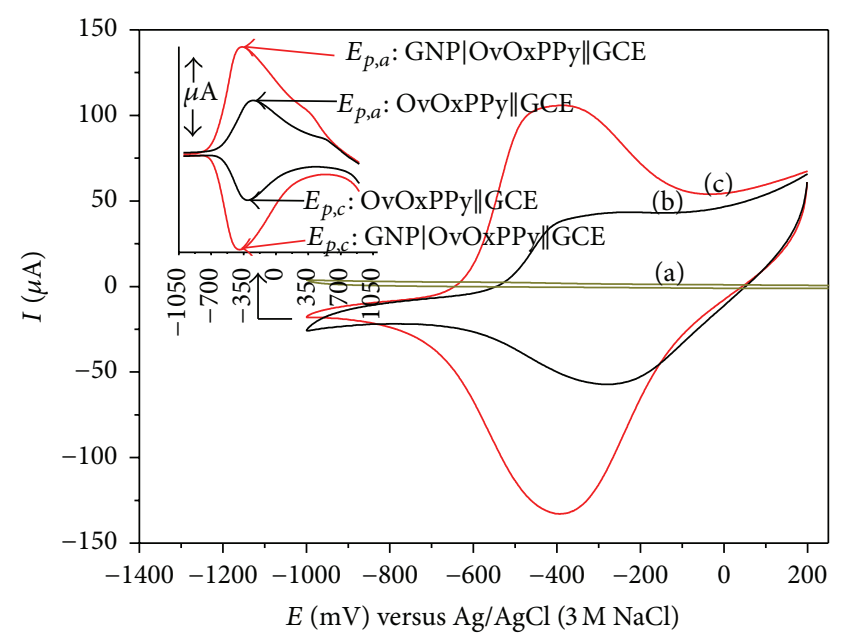

(a) Bare GCE in PBS

(b) OvOxPpy\|GCE in PBS

(c) GNP|OvOxPpy\|GCE in PBS

Figure 3: Cyclic voltammogram of GCE (a), OvOxPpy|lGCE (b), and GNP|OvOxPpyl|GCE (c), in PBS (pH 7.4) at $70 \mathrm{mV} / \mathrm{s}$ versus $\mathrm{Ag} / \mathrm{AgCl}$ (inset): SWV of OvOxPpyl|GCE and the GNP|OvOxPpy||GCE composite film.

ions, takes place, resulting in loss of the conjugated structure and electrical conductivity. Expulsion of the $\mathrm{ClO}_{4}{ }^{-}$dopant ions results in the uniformly sized nanopores within the polymer film.

3.2. Electron Transfer Systems. The modification of the glassy carbon electrode during the film assembly was electrochemically characterized by cyclic voltammetry and square wave voltammetry. From Figure 3, it is evident that the bare glassy carbon electrode shows no observable electrochemistry whereas the background current of the GNP|OvOxPpy||GCE composite is two orders of magnitude higher as compared to that of the overoxidized-polypyrrole film. Moreover, the forward and reverse square waves, in the inset, exhibit a similar trend with regard to magnitude of the background current of GNP|OvOxPpy||GCE composite film as compared to that of the OvOxPpy||GCE film, and, as such, corroborate the findings shown in cyclic voltammetric results.

3.3. Effect of Cobalt(III) Sepulchrate Trichloride. As stated in previous sections, all of the electrochemical studies pertaining to the fabricated CYP3A4-based biosensor were conducted in the presence of diffusional electron transfer mediator, $\mathrm{Co}(\mathrm{Sep})^{3+}$. The main requirement for a good mediator is that it exhibits fast reversible electrochemistry, while also showing a redox response sufficiently negative in order to in turn reduce the enzyme heme group. Prior investigations have established $\mathrm{Co}(\mathrm{Sep})^{3+}$ as superior choice to facilitate electrical communication between redox centres of P450 enzymes and the electrode [24]. Figure 4(a) shows the $\mathrm{CV}$ taken at $100 \mathrm{mV} / \mathrm{s}$ and $\mathrm{SWV}$ recorded at a frequency of $5 \mathrm{~Hz}$. This revealed that as the potential was scanned from -200 to $-800 \mathrm{mV}$, the reduction current steadily increased to a maximum value, after which the current decreased until the switching potential-cathodic and anodic peak currents $\left(I_{p, c}\right.$ and $\left.I_{p, a}\right)$ shown to be fairly similar in magnitude. The CV exhibited well defined corresponding cathodic $\left(E_{p, c}\right)$ and anodic peaks $\left(E_{p, a}\right)$, for which peak potentials were determined as $-650-( \pm 5)$ and $-589( \pm 5) \mathrm{mV}$, respectively, with corresponding $E^{\circ}$, determined as $620 \pm 5 \mathrm{mV}$. The peak potential difference $(\Delta E)$ determined from $C V$ results was approximately $61 \pm 5 \mathrm{mV}$. Square wave voltammetry was used to corroborate CV results. The SW in Figure 4(b) shows well defined forward (cathodic) and reverse (anodic) square waves, with $I_{p, c}$ shown to be slightly higher than $I_{p, a}$, by approximately half an order of magnitude. The peak potentials corresponded to values of -631 and $-620 \pm$ $5 \mathrm{mV}$ for cathodic and anodic square waves, respectively. In comparing $\mathrm{CV}$ and SW results, it is well known that square wave voltammetry has a higher signal-to-noise ratio, and, as such, differences are considered well within experimental variability.

3.4. Monooxygenation of CYP3A4. Figure 5 shows the cyclic voltammograms of the biosensor containing the immobilized redox species at different scan rates, varying from 10 to $100 \mathrm{mV} / \mathrm{s}$. The inset shows the linear variation of anodic and cathodic peak currents $\left(I_{p, a}\right.$ and $\left.I_{p, c}\right)$ with square root of scan rate $\left(v^{1 / 2}\right)$. On increasing the scan rates, the biosensor $\mathrm{CV}$ peak currents increased gradually and peak potentials stayed fairly stable concomitantly resulting in little change in peakto-peak separation with each consecutive scan rate change. $I_{p, c}$ and $I_{p, a}$ also increase linearly with $v^{1 / 2}$. Based on these results it may be deduced that the CYP3A4-film was stable and exhibited reversible electrochemistry, constituted by a diffusion-controlled electron transfer process. The diffusion coefficient, $D$ calculated using the Randle Sevcik equation is $2.5 \times 10^{-8} \mathrm{~cm}^{2} \mathrm{~s}^{-1}$, where $T=25^{\circ} \mathrm{C}, R=8.314 \mathrm{~J} \mathrm{~mol}^{-1} \mathrm{~K}^{-1}, F=$ 


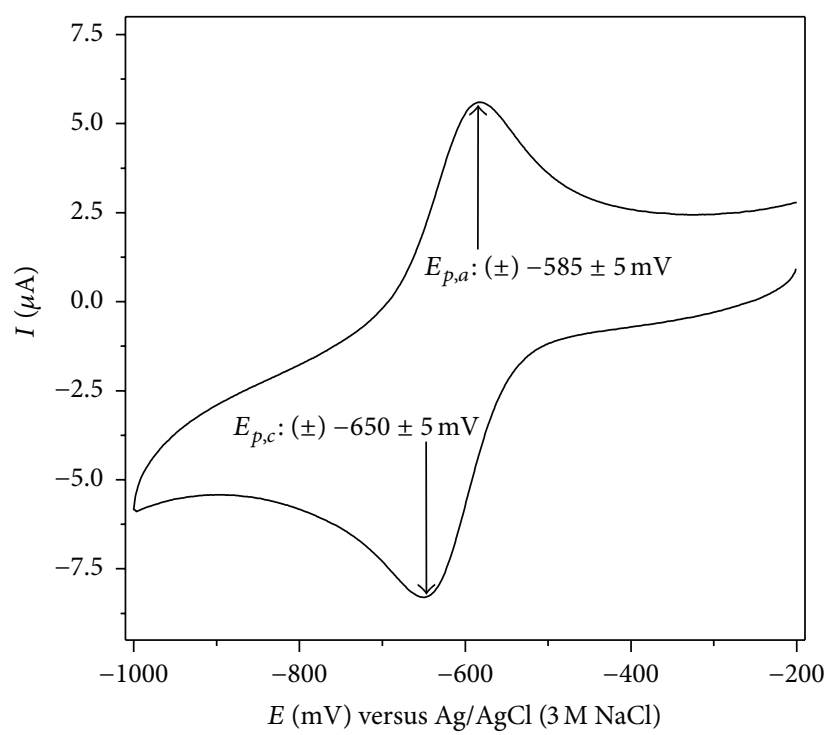

(a)

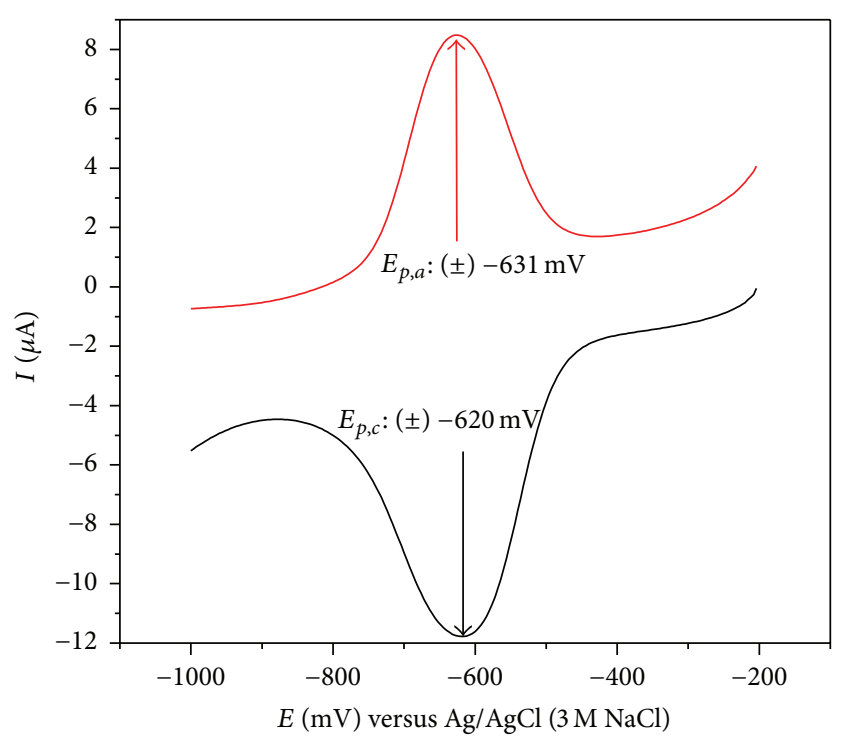

(b)

FIGURE 4: Cyclic (a) and square wave (SW) (b) voltammograms of CYP3A4-based biosensor in argon-saturated PBS (pH 7.4, 100 mM KCl) containing $1 \mathrm{mM} \mathrm{Co}(\mathrm{Sep})^{3+}$ in solution. The biosensor contained 1.63 $\mu \mathrm{M}$ CYP3A4.

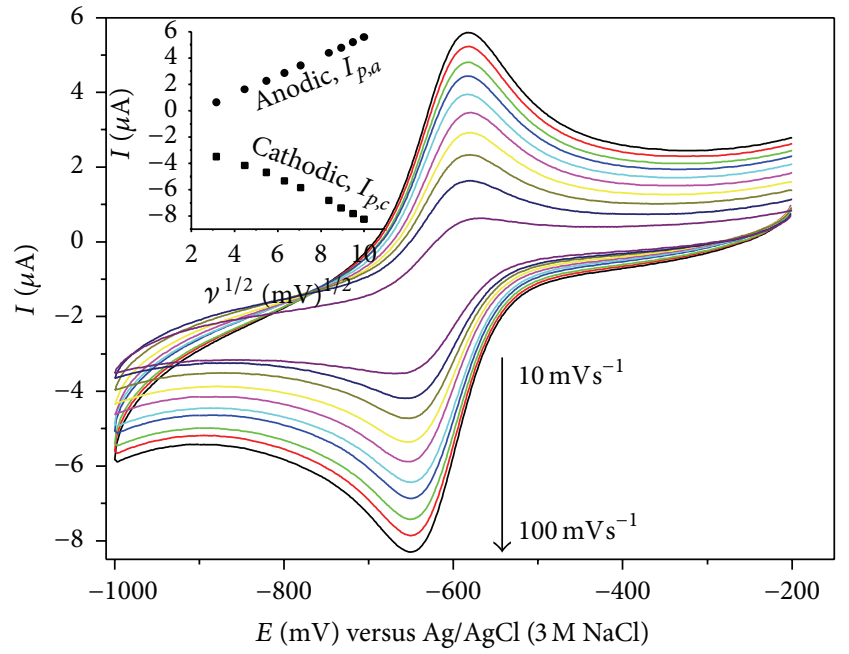

Figure 5: Cyclic voltammograms of biosensor (CYP3A4| GNPs|OvOxPpy||GCE) in argon-saturated PBS (50 mM, $100 \mathrm{mM}$ $\mathrm{KCl})$ containing $1 \mathrm{mM} \mathrm{CoSep}{ }^{3+}$.

$96486 \mathrm{C} \mathrm{mol}^{-1}, C=1 \times 10^{-5} \mathrm{~mol} \mathrm{~cm}{ }^{-3}, n=1, A=0.071 \mathrm{~cm}^{2}$, and slope $=4.67 \times 10^{-3}$ :

$$
\begin{aligned}
& I_{p} \\
& \quad=0.4463 n F A\left[\frac{n F}{R T}\right]^{1 / 2} D^{1 / 2} v^{1 / 2} C_{\text {analyte }} .
\end{aligned}
$$

Concomitantly, the surface concentration, $\Gamma^{*}$ $\left(\mathrm{mol} \mathrm{cm}{ }^{-2}\right)$, of the adsorbed electroactive species may be estimated accordingly:

$$
\frac{I_{p, a}}{v}=\frac{n^{2} F^{2} A \Gamma^{*}}{4 R T},
$$

where $R, F, T$, and $n$ are the gas constant, Faraday constant, temperature, and number of electrons transferred, respectively. The ion exchange behaviour of conducting polypyrrole is associated by a one-electron transfer process in which the monomers are oxidized by oxidizing agents or catalysts to produce the radical polymer cation. Thus, for the one electron transfer process at $25^{\circ} \mathrm{C}, \Gamma^{*}$ value evaluated from the slope of the anodic peak is $6.99 \times 10^{-7} \mathrm{~mol} \mathrm{~cm}{ }^{-2}$.

3.5. Mechanism of Biosensor Response to Indinavir. Voltammetric responses of the biosensor were recorded at a scan rate of $10 \mathrm{mV} / \mathrm{s}$ in the absence and presence of oxygen $\left(\mathrm{O}_{2}\right)$ and/or Indinavir, in order to observe enzyme-oxygensubstrate interactions, and are shown in Figures 6(a), 6(b), and $6(\mathrm{c})$, respectively. In the presence of $\mathrm{O}_{2}$, there was a significant increase in $I_{p, c}$. This is a common phenomenon which has been recorded in literature reports with regard to biosensor studies with $\mathrm{P} 450$ [25]. $\mathrm{O}_{2}$ is the natural cosubstrate of CYP3A4 and P450 enzymes in general and binds to the ferrous ion of the enzyme heme group at a very fast rate; therefore the distinctive $I_{p, c}$ increase can be regarded as a chemical reaction which immediately follows $\mathrm{Fe}^{3+} / \mathrm{Fe}^{2+}$ electron transfer process, a phenomenon which in this case may be described as the electrocatalytic response of the enzyme to $\mathrm{O}_{2}$. The $\mathrm{O}_{2}$-CYP3A4 interaction is greatly enhanced in the presence of substrate and, as such, in the presence of Indinavir $(2.5 \mu \mathrm{M})$ there is a strong amplification in $I_{p, c}$. 


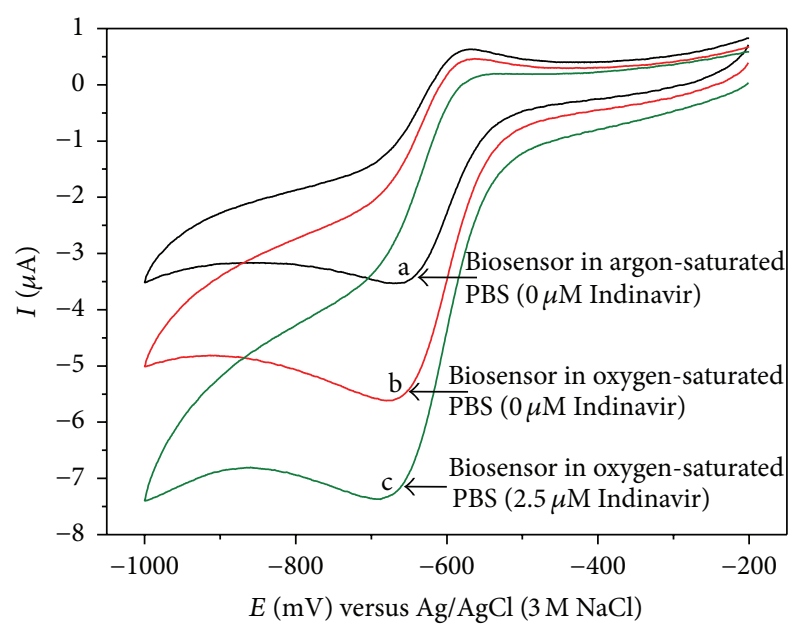

FIGURE 6: Biosensor in argon-saturated PBS ( $0 \mu \mathrm{M}$ Indinavir) (a); oxygen-saturated PBS ( $0 \mu \mathrm{M}$ Indinavir) (b); and oxygen-saturated PBS containing $2.5 \mu \mathrm{M}$ Indinavir (c).

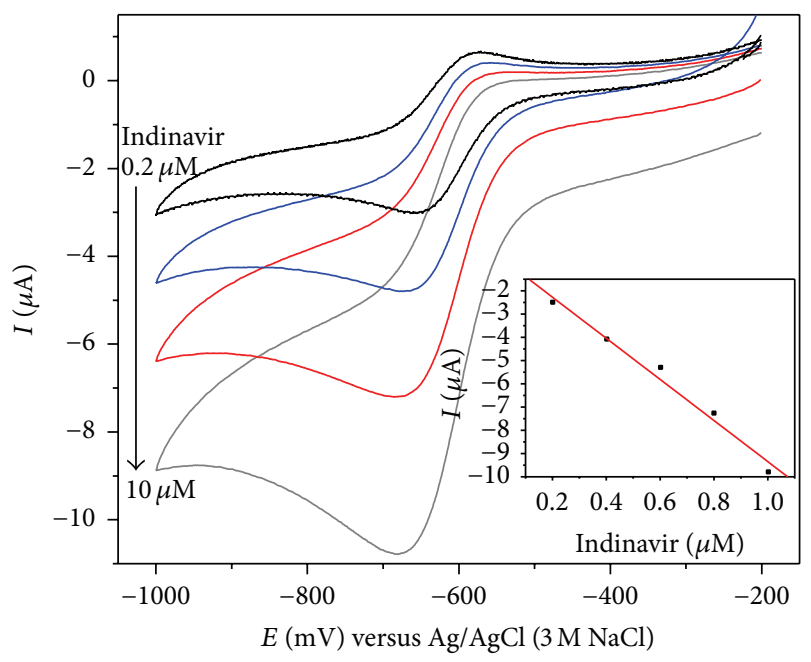

FIGURE 7: CYP3A4-based biosensor in the presence of increasing Indinavir concentrations $(0.2$ to $10 \mu \mathrm{M})$. Inset: cathodic peak current versus Indinavir concentration.

Figure 7 shows the voltammetric response of the biosensor in oxygen-saturated PBS ( $\mathrm{pH} 7.4,100 \mathrm{mM} \mathrm{KCl}$ ) containing $1 \mathrm{mM} \mathrm{Co}(\mathrm{Sep})^{3+}$ diffusional mediator, at a scan rate of $10 \mathrm{mV} / \mathrm{s}$. The CVs reveal that the biosensor exhibits a concentration-dependent increase in $I_{p, c}$ with each addition of Indinavir up to the final concentration of $10 \mu \mathrm{M}$. These findings directly correspond with previous investigations $[26,27]$ and, thus, it may be deduced that the substrate, Indinavir, is metabolised by the reduced CYP3A4, which in turn is mediated by the reduced $\mathrm{Co}(\mathrm{Sep})^{2+}$. The increasing rate of dioxygen to the heme in the presence of substrate is exemplified by the increase in the reduction current $\left(I_{p, c}\right)$ as shown in the cyclic voltammograms. The response profile of the biosensor, as shown by CV, is typical of cytochrome P450 enzyme in particular and heme-enzyme in general, thereby adequately proving the strong catalytic effect and metabolism of Indinavir by CYP3A4. These favourable features of the modified electrode offer great promise for its sensing applications. The inset shows that the catalytic current is linear versus Indinavir concentration in the range of $0.2-10 \mu \mathrm{M}$ and a correlation coefficient of 0.9908 . The detection limit (signalto-noise ratio is 3) is $0.001 \mu \mathrm{mol} \mathrm{L}$. The detection limit is well below the plasma concentration of Indinavir $(8 \mathrm{~h}$ after intake), which ranges from 0.13 to $8.6 \mathrm{mg} \mathrm{L}^{-1}$.

\section{Conclusions}

An ideal nanocomposite support material consisting of GNP infused OvOxPpy was successfully constructed based on a rapid electrochemical technique. The obtained platform served as an ideal host for enzyme immobilization for the development of a user-friendly and low-cost biosensor for Indinavir. The results demonstrated that $\mathrm{Au}$ nanoparticles enhanced the catalytic activity of OvOxPpy by giving negative shifts of CV peak potentials and increasing peak current of the enzyme. Due to the synergy of OvOxPpy and the GNPs, GNP|OvOxPpy||GCE had good stability, sensitivity, and selectivity to determine Indinavir in a large presence of PBS ( $\mathrm{pH}$ 7.21). The biosensor response to Indinavir in oxygenated PBS also showed significant increase. GNP|OvOxPpy||GCE displayed strong catalytic activity towards the oxidation of Indinavir and reveals the response of the active species with distinctive voltammetric peaks. Low concentrations' detection of Indinavir demonstrates the ability of biosensor platform to perform effective point-of-care diagnostics. The biosensor construction method developed in this study, holds great promise for ARV drugs' (Indinavir) determination in real samples and use for effective therapeutic drug monitoring in real time.

\section{Conflict of Interests}

The authors declare that there is no conflict of interests regarding the publication of this paper.

\section{Acknowledgment}

This research was financially supported by South Africa's National Research Foundation (NRF).

\section{References}

[1] N. A. Thompson, "Latin Post," 2015, http://www.latinpost .com/articles/47066.

[2] H. F. Günthard, J. A. Aberg, J. J. Eron et al., "Antiretroviral treatment of adult HIV infection: 2014 recommendations of the International Antiviral Society-USA panel," The Journal of the American Medical Association, vol. 312, no. 4, pp. 410-425, 2014.

[3] H. Namme Luma, M.-S. Doualla, S.-P. Choukem et al., "Adverse drug reactions of Highly Active Antiretroviral Therapy (HAART) in HIV infected patients at the General Hospital, Douala, Cameroon: a cross sectional study," The Pan African Medical Journal, vol. 12, p. 87, 2012. 
[4] V. Perrone, D. Cattaneo, S. Radice et al., "Impact of therapeutic drug monitoring of antiretroviral drugs in routine clinical management of patients infected with human immunodeficiency virus and related health care costs: a real-life study in a large cohort of patients," ClinicoEconomics and Outcomes Research, vol. 6, no. 1, pp. 341-348, 2014.

[5] A. I. Cederbaum, "Molecular mechanisms of the microsomal mixed function oxidases and biological and pathological implications," Redox Biology, vol. 4, pp. 60-73, 2015.

[6] R. Fasan, "Tuning P450 enzymes as oxidation catalysts," ACS Catalysis, vol. 2, no. 4, pp. 647-666, 2012.

[7] D.-H. Zhang, L.-X. Yuwen, and L.-J. Peng, "Parameters affecting the performance of immobilized enzyme," Journal of Chemistry, vol. 2013, Article ID 946248, 7 pages, 2013.

[8] M. Rahman, X.-B. Li, N. S. Lopa, S. J. Ahn, and J.-J. Lee, "Electrochemical DNA hybridization sensors based on conducting polymers," Sensors, vol. 15, no. 2, pp. 3801-3829, 2015.

[9] Z. Wang, N. Sun, Y. He, Y. Liu, and J. Li, "DNA assembled gold nanoparticles polymeric network blocks modular highly sensitive electrochemical biosensors for protein kinase activity analysis and inhibition," Analytical Chemistry, vol. 86, no. 12, pp. 6153-6159, 2014.

[10] F. Valentini and G. Palleschi, "Nanomaterials and analytical chemistry," Analytical Letters, vol. 41, no. 4, pp. 479-520, 2008.

[11] Y. Okawa, N. Yokoyama, Y. Sakai, and F. Shiba, "Direct electron transfer biosensor for hydrogen peroxide carrying nanocomplex composed of horseradish peroxidase and $\mathrm{Au}-$ nanoparticle-characterization and application to bienzyme systems," Analytical Chemistry Research, vol. 5, pp. 1-8, 2015.

[12] W. Putzbach and N. J. Ronkainen, "Immobilization techniques in the fabrication of nanomaterial-based electrochemical biosensors: a review," Sensors, vol. 13, no. 4, pp. 4811-4840, 2013.

[13] H. Mallin, J. Muschiol, E. Byström, and U. T. Bornscheuer, "Efficient biocatalysis with immobilized enzymes or encapsulated whole cell microorganism by using the SpinChem reactor system," Chem CatChem, vol. 5, no. 12, pp. 3529-3532, 2013.

[14] R. A. Sheldon, M. J. Sorgedrager, and M. H. A. Janssen, "Use of cross-linked enzyme aggregates (CLEAs) for performing biotransformations," Chemistry Today, vol. 25, pp. 62-67, 2007.

[15] N. R. Mohamad, N. H. Marzuki, N. A. Buang, F. Huyop, and R. A. Wahab, "An overview of technologies for immobilization of enzymes and surface analysis techniques for immobilized enzymes," Biotechnology \& Biotechnological Equipment, vol. 29, no. 2, pp. 205-220, 2015.

[16] P. Zucca and E. Sanjust, "Inorganic materials as supports for covalent enzyme immobilization: methods and mechanisms," Molecules, vol. 19, no. 9, pp. 14139-14194, 2014.

[17] I. Ince, C. A. J. Knibbe, M. Danhof et al., "A novel maturation function for clearance of the cytochrome P450 3A substrate midazolam from preterm neonates to adults," Clinical Pharmacokinetics, vol. 52, no. 7, pp. 555-565, 2013.

[18] K. Ariga, Q. Ji, T. Mori et al., "Enzyme nanoarchitectonics: organization and device application," Chemical Society Reviews, vol. 42, no. 15, pp. 6322-6345, 2013.

[19] L. Sasso, A. Heiskanen, F. Diazzi et al., "Doped overoxidized polypyrrole microelectrodes as sensors for the detection of dopamine released from cell populations," Analyst, vol. 138, no. 13, pp. 3651-3659, 2013.

[20] B. G. Kalzung, S. B. Masters, and A. J. Trevor, Eds., Basic \& Clinical Pharmacology, McGraw-Hill Medical, New York, NY, USA, 11th edition, 2010.
[21] A. Ignaszak, N. Hendricks, T. Waryo et al., "Novel therapeutic biosensor for indinavir-a protease inhibitor antiretroviral drug," Journal of Pharmaceutical and Biomedical Analysis, vol. 49, no. 2, pp. 498-501, 2009.

[22] M. Björninen, A. Siljander, J. Pelto et al., "Comparison of chondroitin sulfate and hyaluronic acid doped conductive polypyrrole films for adipose stem cells," Annals of Biomedical Engineering, vol. 42, no. 9, pp. 1889-1900, 2014.

[23] A. Fakhry, H. Cachet, and C. D. Chouvy, "Electrochemical characterisations of ultra thin overoxidized polypyrrole films obtained by one-step electrosynthesis," Journal of the Electrochemical Society, vol. 160, no. 10, pp. D465-D470, 2013.

[24] E. A. Schneider, Oriented attachment of cytochrome P450 2C9 to a self-assembled monolayer on a gold electrode as a biosensor design [Ph.D. thesis], University of California, San Francisco, Claif, USA, 2011.

[25] C. Baj-Rossi, G. De Micheli, and S. Carrara, "Electrochemical detection of anti-breast-cancer agents in human serum by cytochrome p450-coated carbon nanotubes," Sensors, vol. 12, no. 5, pp. 6520-6537, 2012.

[26] N. R. Hendricks, T. T. Waryo, O. Arotiba, N. Jahed, P. G. L. Baker, and E. I. Iwuoha, "Microsomal cytochrome P4503A4 (CYP3A4) nanobiosensor for the determination of 2,4dichlorophenol-an endocrine disruptor compound," Electrochimica Acta, vol. 54, no. 7, pp. 1925-1931, 2009.

[27] E. I. Iwuoha, R. Ngece, M. Klink, and P. Baker, "Amperometric responses of CYP2D6 drug metabolism nanobiosensor for sertraline: a selective serotonin reuptake inhibitor," IET Nanobiotechnology, vol. 1, no. 4, pp. 62-67, 2007. 

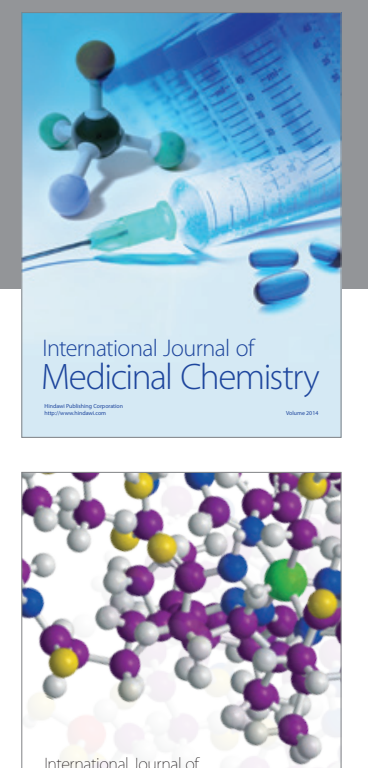

\section{Carbohydrate} Chemistry

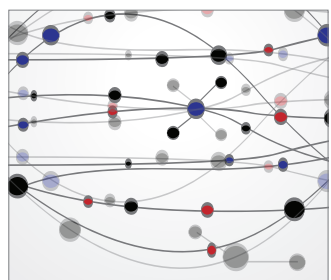

The Scientific World Journal
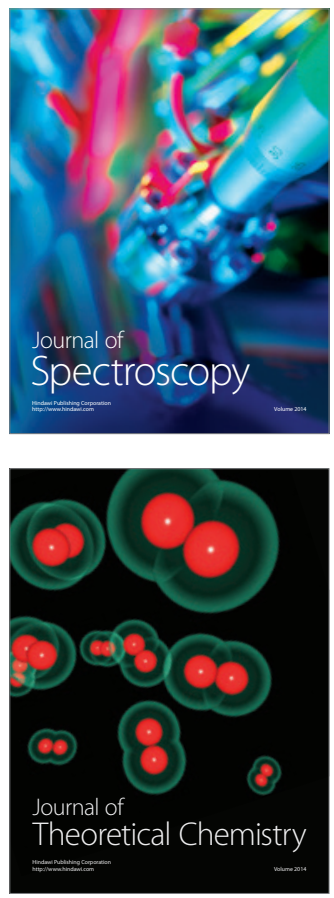
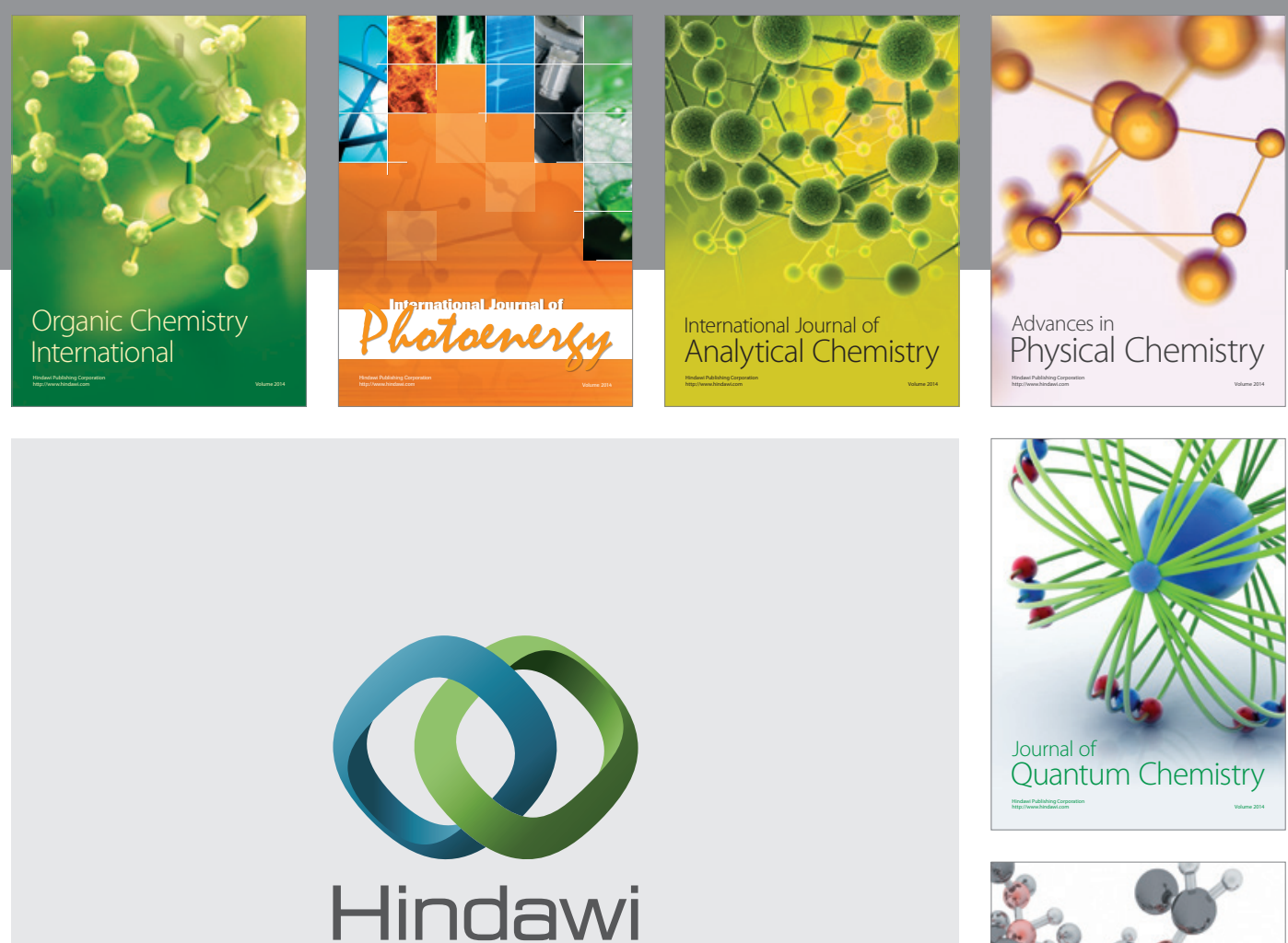

Submit your manuscripts at

http://www.hindawi.com

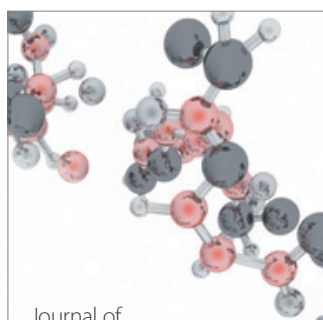

Analytical Methods

in Chemistry

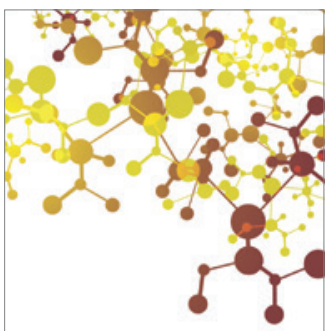

Journal of

Applied Chemistry

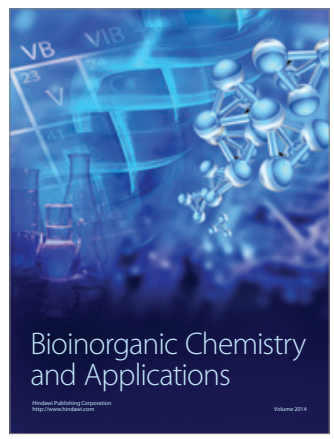

Inorganic Chemistry
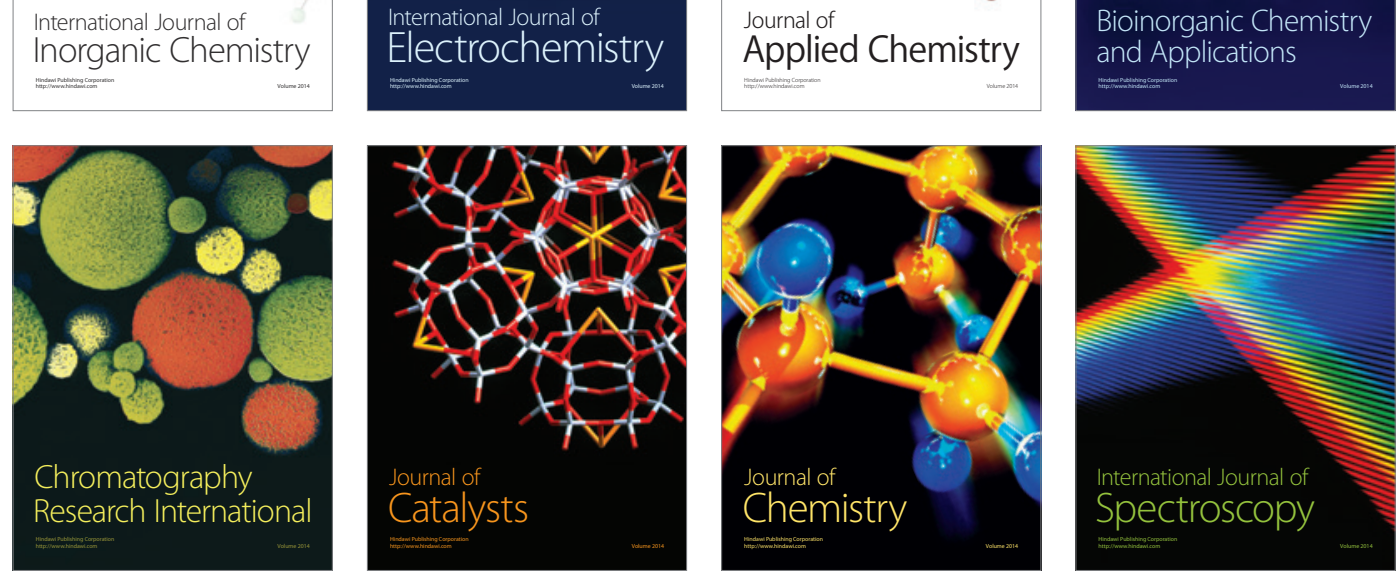